\title{
Assessing the performance of commercial Agisoft PhotoScan software to deliver reliable data for accurate 3D modelling
}

\author{
Ahmed Jebur ${ }^{1, *}$, Fanar Abed ${ }^{2}$ and Mamoun Mohammed ${ }^{1}$ \\ ${ }^{1}$ Engineering Technical College, Central Technical University, Baghdad, Iraq \\ ${ }^{2}$ College of Engineering, University of Baghdad, Baghdad, Iraq
}

\begin{abstract}
D models delivered from digital photogrammetric techniques have massively increased and developed to meet the requirements of many applications. The reliability of these models is basically dependent on the data processing cycle and the adopted tool solution in addition to data quality. Agisoft PhotoScan is a professional image-based 3D modelling software, which seeks to create orderly, precise $\mathrm{n}$ 3D content from fixed images. It works with arbitrary images those qualified in both controlled and uncontrolled conditions. Following the recommendations of many users all around the globe, Agisoft PhotoScan, has become an important source to generate precise 3D data for different applications. How reliable is this data for accurate 3D modelling applications is the current question that needs an answer. Therefore; in this paper, the performance of the Agisoft PhotoScan software was assessed and analyzed to show the potential of the software for accurate 3D modelling applications. To investigate this, a study was carried out in the University of Baghdad / Al-Jaderia campus using data collected from airborne metric camera with $457 \mathrm{~m}$ flying height. The Agisoft results show potential according to the research objective and the dataset quality following statistical and validation shape analysis.
\end{abstract}

\section{Introduction}

Photogrammetry is a suitable tool to equip information about man-made and natural objects such as terrain, vegetation and land cover urban features. Photogrammetry and digital image-based processing techniques have an important role to make visualization technology practical and provide low cost workflow when compared to traditional and geodetic survey. The photogrammetric process offers various benefits over the traditional land surveying methods[1]. It represents the elastic job which any project data requires and can be accomplished at the same time it would be long lasting, and corresponding with the permanent cost of image acquisition. It can also be supported with data of higher resolution for digital mapping products such as maps; digital elevation models (DEMs) and orthoimages. Due to this capability, it is convenient and practical for many applications, such as archaeology, monitoring issues and 3D city modelling [2].

A 3D city model is a digital representation of an urban environment with 3D geometry of urban features and structures, however, buildings have the most feature print phenomenon [3]. 3D city models can be technically generated from different digital techniques such as photogrammetry, laser scanning and others. 3D city model has become ready and available for several purposes beyond visualization, and are used in a large number of fields including Engineering [4]. The 3D city model, simulation is done by using true and reliable information via photogrammetry which can be provided with the only economic means to obtain truly $3 \mathrm{D}$ city data [5]. With regards to precision and accuracy, 3D city models can be used for simulation and analysis from visualization and animation $[6,7]$.

Reference [7] discuss about using the Leica Photogrammetric Suite (LPS) software to deliver 3D data in order to create (DTM) from Aerial Photographs. Reference [8] discuss about 3D building modelling from Close range Photogrammetry by using PhotoModeler scanner software and found to be a reliable software for accurate derivable for short-range images. However, [9] also discuss about the use of digital camera for Close Range Photogrammetry (CRP) by using PhotoModeler scanner software. Their result shows that CRP technique is capable to generate the $3 \mathrm{D}$ photorealistic model and structural building with photo texturing. On the other hand, [10] introduces a modelling method from aerial images for 3D city modelling. They used a commercial photogrammetric tool and deliver promising outputs showing the validity in terms of time, labor, and reusability. Further, [11] discuss the production of 3D city model for Al-Yarmouk University by using Photogrammetry techniques and geographic information system (GIS). In this study they have discussed the corresponding required production workflow for building 3D GIS Model of a complex site of interest using Digital Photogrammetry.

Corresponding author: kareemahmed1980@yahoo.com 
In a 3D city model development, it is important to choose suitable data with high reliability, appropriate methods and high-efficiency software for production [9]. These data can be derived from aerial images, terrestrial photos and laser scanning data etc. However, the highefficiency software such as (LPS) Leica photogrammetry suite, Agisoft Photoscan or PhotoModeler scanner or others, has to be investigated and validated for individual data. As the majority of the research depends on various softwares, this research discusses the possibility of using the Agisoft Photoscan software to deliver reliable data for accurate $3 \mathrm{D}$ modelling applications. It also features other different methods to evaluate the results obtained through the Agisoft Photoscan software, compared to the Leica photogrammetry suite (LPS) which has been referred to in this work.

\section{Study Area and Dataset}

The study area comprises the boundaries of the college of Engineering / University of Baghdad / IRAQ which equals to (1.33329 sq. $\mathrm{km})$. It is located within $\left(33^{\circ} 16^{\prime}\right.$ -

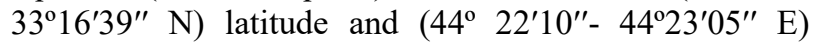
longitude, respectively as shown in Figure (1).

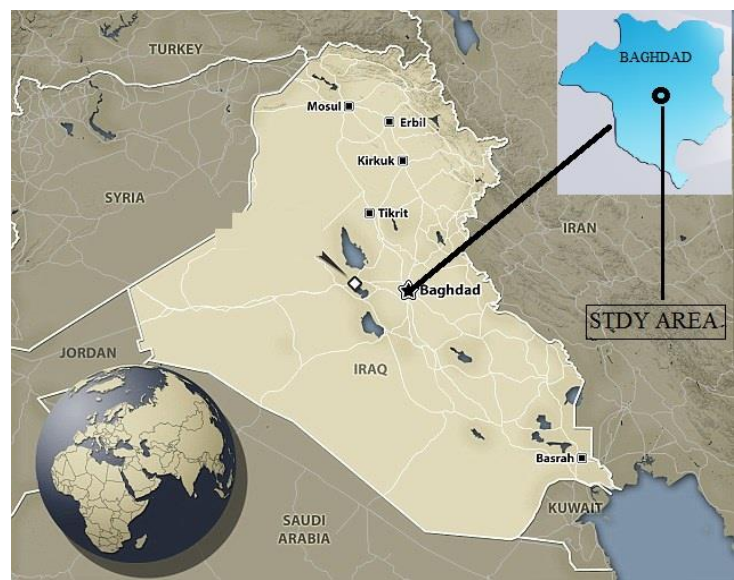

Fig.1. Study Area.

The dataset consists of eight aerial photos captured by metric camera format of $(230 \times 230 \mathrm{~mm})$ in 1983 . This dataset is an interesting one to be investigated because it was the first photogrammetry project to cover the majority of Baghdad University. The panchromatic images are consisting of two flight lines with $20 \%$ side lap and more than $60 \%$ end lap. These images were captured with flying height of $457 \mathrm{~m}$ above ground and camera focal length of $152.16 \mathrm{~mm}$ as shown in Figure (2).

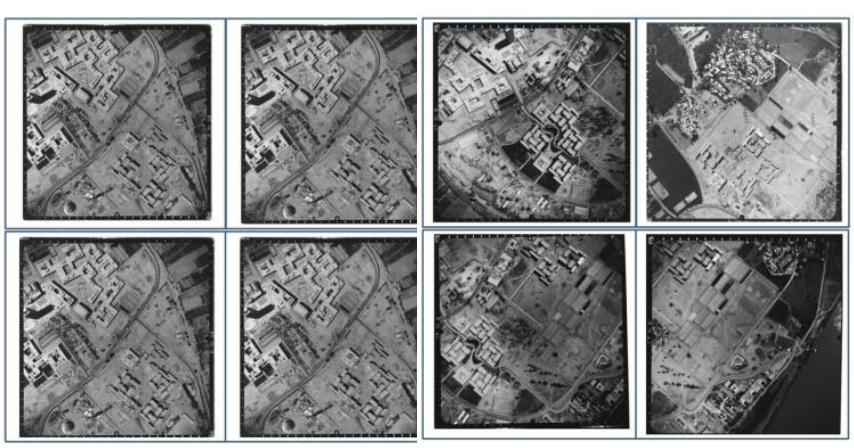

Fig.2. Represents The Block of Photos of The Dataset Including Flight lines 1 and 2.

\section{Methodologies}

This research was carried out in four basic stages. The first stage was accomplished by collecting GCPs and adjusting the network delivered. The second stage consisted of photo processing and triangulation using least squares solution. The third stage consisted of implemented procedures to carry out the spatial analysis to create raster surface. And the last stage included the statistical analysis, assessment, and validation of the generated 3D surface.

\subsection{Ground Control Points (GCPs) Collection}

The ground control points were obtained by using differential global positioning system (DGPS) technique, and later adjusted accurately following robust least squares scenario. The type of the GPS device used to collect the data was Topcon GR-5, and the working mode was the real time kinematic (RTK). The coordinates of 29 ground points were collected and located on well-defined features with good and homogeneous distribution over the study area. These were measured with reference to the UTM projection reference. From all collected points, only 20 were used as GCPs and the remaining 9 points were used as Check Points as show in Figure (3).

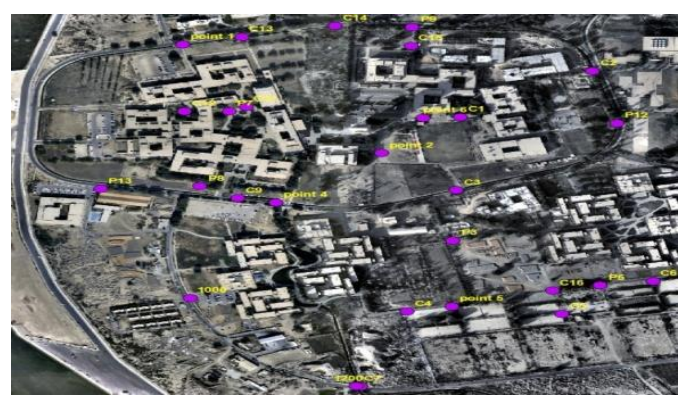

Fig.3. Distribution of GCPs and Check Points in Study Area.

A high number of Check Points were used in this research because the reliability of the accuracy estimations depends among other variables, on the 
number of Check Points and their distribution [12]. Later a network of $3 \mathrm{D}$ coordinates are delivered, however, it cannot be post-processed for accurate later stages without adjustment. Therefore, 3D network adjustment was implemented to adjust all of the 38 GCPs using nonlinear solution (variation of coordinate's model). Least squares routine was adopted for accurate final derivable. The adjusted coordinates of the GCPs was used later on for the next stage of processing.

\subsection{Photo Processing by Simultaneous Solution}

\section{(Bundle Adjustment)}

The photo processing with aerotriangulation workflow is also named as bundle adjustment technique because it is based on the idea that many light rays which come from the light source (camera sensor) are forming a bundle of rays all intersects to generate spatial positions. The bundles from all involved images are later adjusted simultaneously so that corresponding light rays intersect at positions of ground features [13]. Collinearity equations are the formula used as a mathematical model to adjust these bundles using least squares. However, these equations are not linear and thus, need to be linearized for an optimal solution. This can be obtained mathematically by adopting the first order terms of Taylor's theorem using initial values to the unknowns [14]. 3D dense point clouds are generated from this step as input for the next further steps.

\subsubsection{Bundle Block Adjustment using LPS software}

Leica Photogrammetry Suite (LPS), provided a flexible environment for aeritriangulation and bundle adjustment. LPS is a commercial digital photogrammetric solution which allows fast processing of many types of imagebased solutions such as triangulation or orthorectification with accurate and precise outputs. It can manipulate any kind of image collected from different sources of cameras and satellite sensors. It can easily model various camera and sensor types by generating the image geometry at the time of exposure and deliver the orientation parameters for individual images at the time of collection. Later, the software computes the mathematical relationship between the bundle of images to compute the geospatial coordinates of the ground points based on the collinearity condition equations assumption [15]. This can be applied by following space intersection and space resection techniques for the bundle of images simultaneously. However, to do so it is important to engage some additional techniques during the process of image capturing, these are the GPS and the INS techniques. They both normally deliver initial measurements to the exterior orientation; however, these measurements need to be adjusted for further processing in the triangulation modelling due to high level accuracy requirement [15]. A bundled solution is adopted to calculate the exterior orientation parameters of individual images in the block and the final geospatial coordinates of the unknown points. The block of eight images (2 flight lines) was included in this research and was simultaneously treated in one solution. A least squares adjustment is applied to compute the unknowns in the entire block following multi iterations to decrease individual errors. It is obvious that block triangulation is the optimal operation to define the mathematical relationship between the images in a certain block and the ground. Once the relationship is defined correctly, accurate geospatial positions of ground features can be extracted.

\subsubsection{Bundle Block Adjustment using Agisoft photoscan software}

Agisoft PhotoScan is professional image-based 3D modeller software seeking at creating orderly precision 3D content from fixed images [16]. It works with arbitrary images those qualified in both controlled and uncontrolled conditions. In Agisof PhotoScan software, the images can be captured from any position, in a way that the object can be reconstructed from at least two photos taken from different positions. All the process including image alignment and 3D model reconstruction are fully automated as shown in Figure (4). Following the recommendations of many users all around the globe, Agisoft PhotoScan, has become an important source to generate precise 3D data for different applications.

Agisoft photoscan Project Manager also uses the bundle block adjustment for triangulation method. However, Agisoft PhotoScan has a major issue regarding photo orientation as compared to the LPS. This becomes obvious when the photos are highly oriented from the Nadir (in Kappa direction). This can be interpreted due to the lack of the PhotoScan software to apply photo calibration including distortion parameters during the adjustment process. This could be a major issue if the photos were not originally in digital form and has been transformed into a digital form through scanning process which is the case in this research.

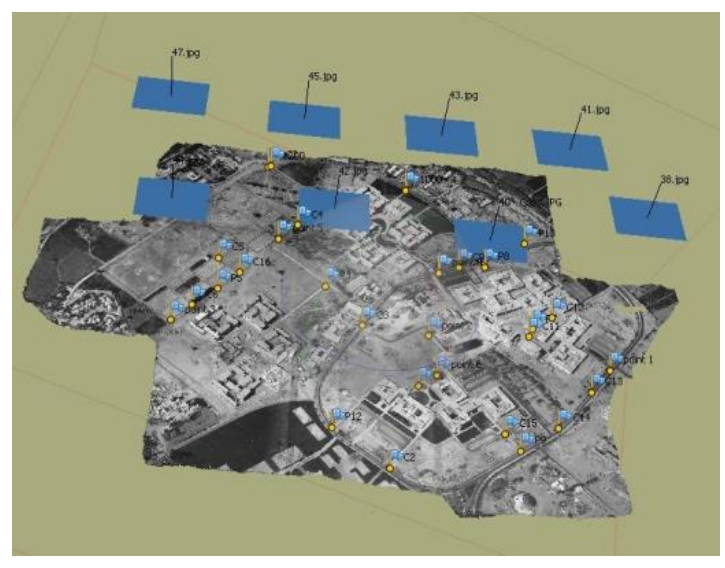

Fig.4. Illustrate Image Alignment and 3D Model Reconstruction.

\subsection{Spatial Analysis (Interpolation)}


Interpolation is the procedure used to foretell cell values in locations that Lack sample points. It is based on the principle of spatial self-correlation or spatial dependence between the near and distant organism. Spatial analyst methods which are considered to be one of the important tools in GIS, these tools are used to create maps. Spatial analysis method is a public term for all manipulations of spatial data with the aim of improving one's understanding of the geographical phenomena represented by the data [17]. In this research the spatial analysis was carried out using the ArcGIS ${ }^{\circ}$ Geostatistical Analyst toolbar to generate 3D model. The spatial analysis was conducted using multiple interpolation methods which are available in the ArcGIS ${ }^{\circledR}$ Geostatistical Analyst toolbar. The spatial interpolators assessed in this work were: kriging method, IDW method, and spline method. These methods were chosen because they are widely used and approved in the literature.

\subsubsection{Spatial Analysis Methods}

\subsubsection{Kriging Method}

Kriging is a geostatistical gridding method that has proved its validity and efficiency in many applications. This method produces visually attractive maps of irregularly spaced data. Kriging is a very flexible gridding method and can produce very accurate surfaces of any kind of data. It also can be custom-fit to a data set, by specifying the appropriate variogram model. Within Arc Map, Kriging can be either a smoothing or an exact interpolator, depending on the specified parameters [18].

\subsubsection{Inverse Distance Weighting (IDW) Method}

The Inverse Distance is a weighted average interpolator, which can be either smoothing or exact interpolate data. Data are weighted with this method to a certain power during the interpolation process, so that the influence of one point, relative to another, decreasing with distance from the node of grid. The greater the power of weighting when far removed from the grid node, lesser effect on the points, during interpolation [19]. By using a linear -weighted combination IDW determines the values of the cells for a set of sample points. The weight assigned is considered as a function of the distance from a certain input point to the output cell location. The greater the distance, lesser the effect on the output value [17].

\subsubsection{Nearest Neighbours Method}

The nearest neighbours $(\mathrm{NN})$ method foresees the value of the attribute at an un sampled point based on known values of the nearest sampled points. This can be achieved by drawing perpendicular bisectors between sampled points (n), forming such as Thiessen polygons. This produces one polygon for individual samples and also in the centre of the polygon located in the sample. All points are nearer to its enclosed sample point than to any other sample points in each polygon [20]. Within each polygon all points (or locations) are assigned the same value (weights) [21].

\subsection{Statistical Analysis and Validation Assessments}

In order to determine the difference between both softwares which have been used, comparison between the produced data from both softwares has been. The method that was used for the comparison is statistical, which depends on some of the standards. In order to assess the surface generated by different interpolation methods, some field measurements has adopted for this assessment, which is regarded as a reference, such as length, width or building area as shown in Figure (5). In order to verify the accuracy and reliability of the surface formed by the methods of interpolation, comparison was made with field measurements.
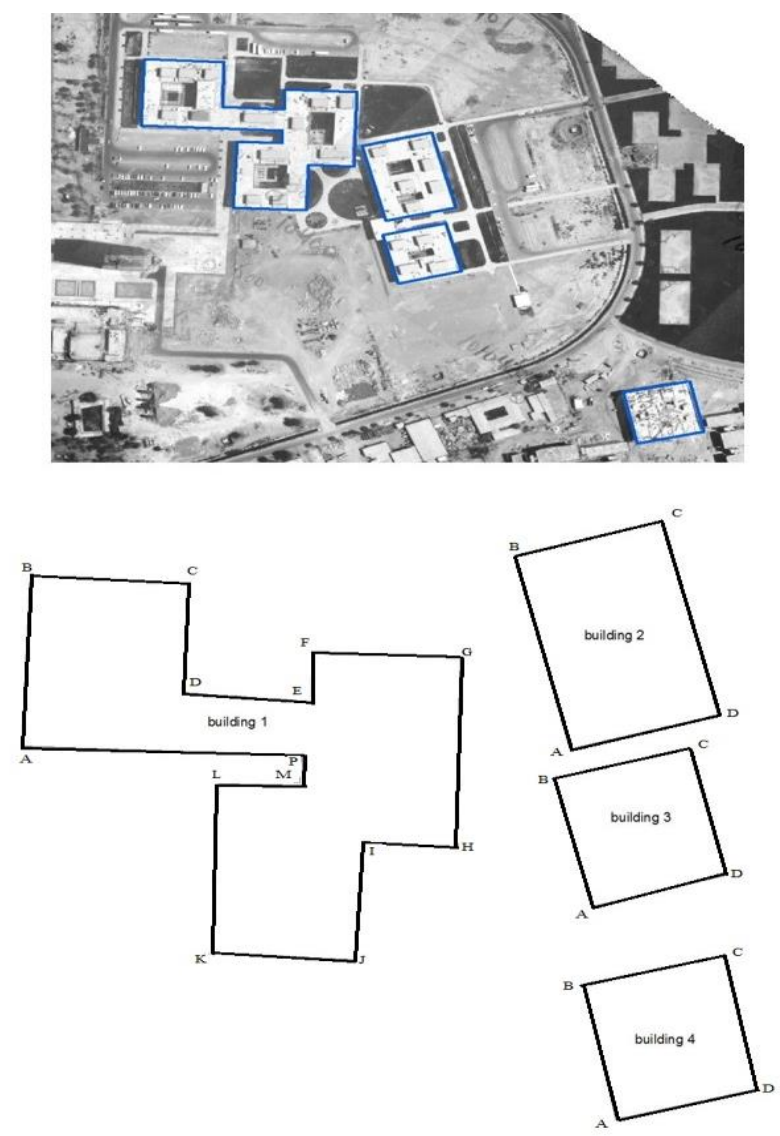

Fig.5. Illustrates Field Measurements for Validation Assessment.

\section{Results}

\subsection{Result of GCPs Adjustment}


Table (1) shows the corrected coordinates of ground control points using least squares method after applying 3D network adjustment as stated in section 3.1.

Table 1. The Corrected 3D Coordinates of the GCPs.

\begin{tabular}{|c|c|c|c|c|c|}
\hline & $\begin{array}{l}\text { Point } \\
\text { ID }\end{array}$ & $\mathbf{X}_{\mathrm{m}}$ & $Y_{m}$ & $\mathbf{Z}_{\mathrm{m}}$ & Type \\
\hline 1 & P7 & 441820.02 & 3681854.52 & 36.12 & Control \\
\hline 2 & Point 2 & 442057.74 & 3681729.71 & 35.63 & Control \\
\hline 3 & Point 6 & 442122.97 & 3681835.03 & 36.60 & Control \\
\hline 4 & $\mathrm{C} 2$ & 442387.34 & 3681975.24 & 35.05 & Control \\
\hline 5 & P12 & 442426.57 & 3681818.94 & 35.54 & Control \\
\hline 6 & P3 & 442169.90 & 3681468.58 & 36.78 & Control \\
\hline 7 & Point 5 & 442168.13 & 3681271.72 & 35.92 & Control \\
\hline 8 & P5 & 442400.08 & 3681334.27 & 36.00 & Control \\
\hline 9 & 1200 & 442024.10 & 3681031.63 & 35.71 & Check \\
\hline 10 & $\mathrm{C} 7$ & 442018.14 & 3681032.84 & 35.72 & Control \\
\hline 11 & C9 & 441833.53 & 3681595.12 & 34.90 & Check \\
\hline 12 & C11 & 441846.08 & 3681868.01 & 36.15 & Check \\
\hline 13 & Point 1 & 441747.75 & 3682054.30 & 33.82 & Control \\
\hline 14 & P9 & 442106.40 & 3682106.20 & 35.01 & Check \\
\hline 15 & $\mathrm{C} 15$ & 442103.99 & 3682051.10 & 35.20 & Check \\
\hline 16 & $\mathrm{C} 13$ & 441840.13 & 3682077.14 & 35.74 & Control \\
\hline 17 & P13 & 441619.08 & 3681622.60 & 34.79 & Control \\
\hline 18 & Point 3 & 442550.16 & 3681361.10 & 35.94 & Control \\
\hline 19 & 1000 & 441759.13 & 3681296.50 & 34.99 & Check \\
\hline 20 & $\mathrm{C} 3$ & 442174.52 & 3681618.74 & 36.51 & Control \\
\hline 21 & C14 & 441986.11 & 3682110.09 & 35.59 & Control \\
\hline 22 & $\mathrm{C} 1$ & 442181.34 & 3681837.57 & 36.89 & Check \\
\hline 23 & $\mathrm{C} 12$ & 441749.52 & 3681854.89 & 36.42 & Control \\
\hline 24 & Point 4 & 441892.90 & 3681582.47 & 35.63 & Control \\
\hline 25 & P8 & 441774.15 & 3681630.71 & 35.41 & Control \\
\hline 26 & $\mathrm{C} 4$ & 442097.56 & 3681256.32 & 36.25 & Control \\
\hline 27 & $\mathrm{C} 5$ & 442339.65 & 3681249.01 & 36.39 & Check \\
\hline 28 & C16 & 442324.72 & 3681318.42 & 36.69 & Control \\
\hline 29 & C6 & 442482.30 & 3681346.87 & 36.10 & Check \\
\hline
\end{tabular}

\subsection{Result and Analysis of The bundle Block Adjustment}

Triangulation has been applied by using LPS and Agisoft photoscan software. In individual overlapped areas at least three GCPs must be specified for each stereo pair, and on stereo image, several tie points were measured. The triangulation method is performed to estimate the ground coordinates $(\mathrm{X}, \mathrm{Y}, \mathrm{Z})$ locations of tie points in stereo models. It also computed the exterior orientation parameters (EOP) of individual images. Figure (6) illustrates the distribution of the GCPs, tie points and check points in the adjusted stereo models. However, Figure (7) illustrates the distribution of the GCPs and check points in the adjusted stereo model by Agisoft photoscan software.

In triangulation, a less number of control points were used in the block for both softwares. The results of the triangulation are 3D point's clouds as shown in Figures ( 8 and 9). The number of the generated point's clouds was equal to 1784 by LPS software points, however, more than 7519294 points was generated by Agisoft photoscan software.

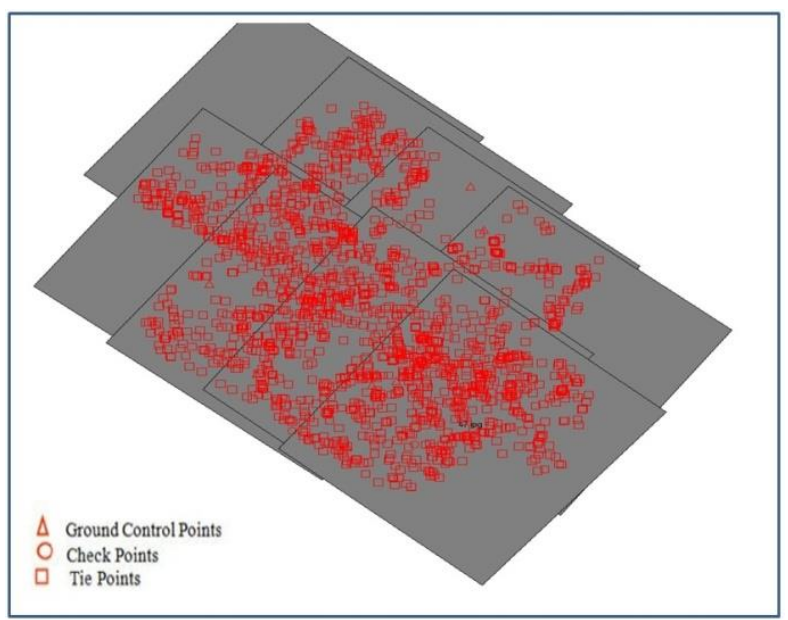

Fig.6. The Distribution of the GCPs, Tie Points and Check Points in the Adjusted Stereo Model by LPS software.

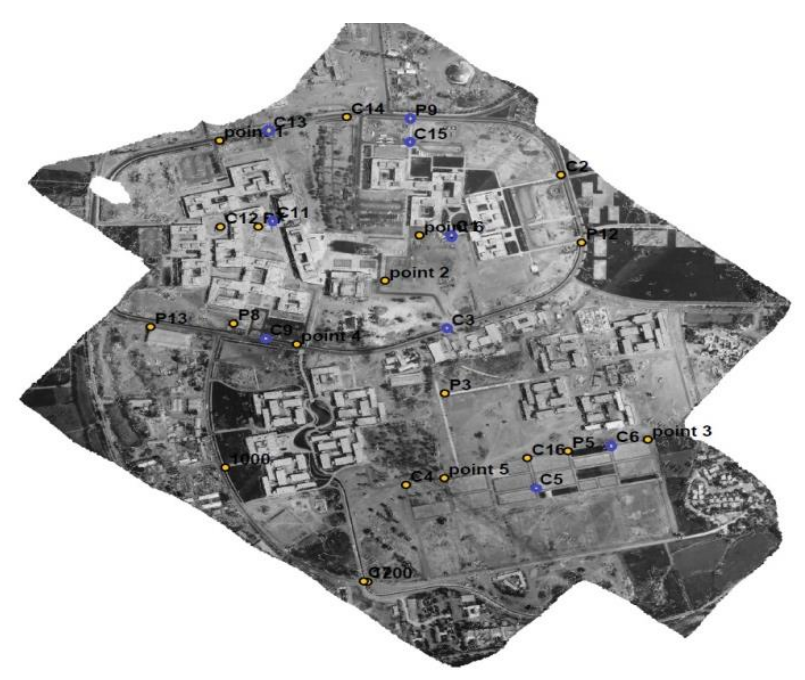

Fig.7. Distribution of Ground Control Points and Check Points in the Adjusted Stereo Model by Agisoft Photoscan software.

After the process of triangulation has been completed, RMSE was calculated. Nine check points were used to compare the triangulated coordinates from photogrammetry with those measured by DGPS and adjusted later. The results are listed in Tables (2 and 3) for both softwares. 


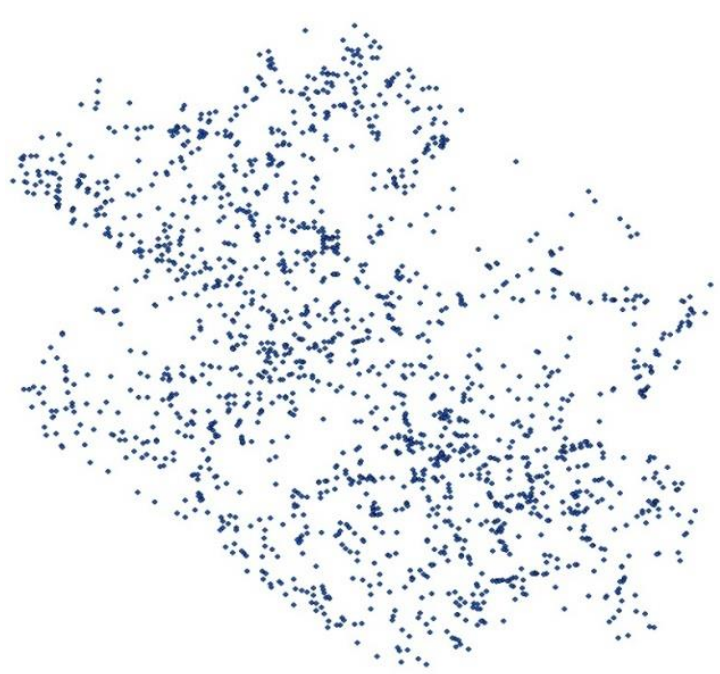

Fig.8. Illustrates the Triangulated Points Clouds Generated From LPS Software.

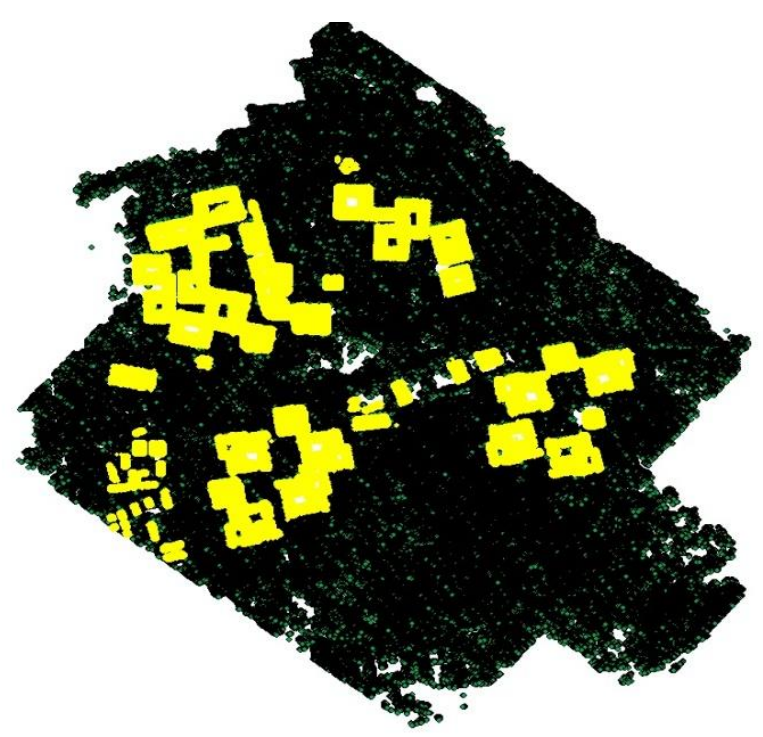

Fig.9. Illustrates the Triangulated Points Clouds Generated From Agisoft Photoscan Software.

\subsection{Result of Interpolation Methods}

For interpolation purposes, points were exported from LPS and Agisoft Photoscan softwares to Arc Map for spatial analysis and to generate a surface for the study area. The results are as shown in Figures (10, 11 and 12) and Figures (13, 14 and 15) for both the softwares, respectively.

\subsection{Result of Statistical Analysis and Validation Assessment}

Statistical analysis was performed on the points clouds that were generated by Agisoft photoscan and LPS software. In this research, statistical analysis was conducted by using one-sample $\mathrm{T}$-test to find error values. One-sample T-test stating an assumption about a population with large sample sizes, generally meaning $\geq$ 30 , from a continuous population, the distribution of sample is well approximated by a normal distribution. For this research there is one value to accept or reject which is the null hypothesis: p-value. Whereas, P-value referred to significance level, it is used as a standard for accepting or rejecting the null hypothesis. For this study, 95\% CI was applied according to NSSDA approach whereas $\mathrm{P}$-value is 0.05 . After finding the value of onesample T-test, the P-value from the statistical table can be easily determined. Further it cannot reject the null hypothesis when the P-value is greater than 0.05 , and it should accept alternative hypothesis when P-value is less than 0.05 . The following Figures (16 and 17) show the results of the statistical analysis. 
Table 2. RMSE Delivered from LPS Software.

\begin{tabular}{|c|c|c|c|c|c|c|}
\hline \multirow[b]{2}{*}{ Point ID } & \multicolumn{3}{|c|}{$\begin{array}{l}\text { Reference coordinates of check Points } \\
(\mathbf{m} .)\end{array}$} & \multicolumn{3}{|c|}{$\begin{array}{l}\text { Calculated coordinates of check Points } \\
\qquad(\mathrm{m} .)\end{array}$} \\
\hline & $\mathbf{X}$ & $\mathbf{Y}$ & $\mathbf{Z}$ & $\mathbf{X}$ & $\mathbf{Y}$ & $\mathbf{Z}$ \\
\hline 1200 & 442024.99 & 3681031.63 & 35.71 & 442024.82 & 3681031.48 & 35.15 \\
\hline C9 & 441833.53 & 3681595.12 & 34.90 & 441833.79 & 3681594.96 & 35.17 \\
\hline $\mathrm{C} 11$ & 441846.08 & 3681868.01 & 36.15 & 441846.12 & 3681867.75 & 36.25 \\
\hline P9 & 442106.40 & 3682106.20 & 35.01 & 442106.36 & 3682106.23 & 34.99 \\
\hline $\mathrm{C} 15$ & 442103.99 & 3682051.10 & 35.20 & 442104.59 & 3682051.16 & 34.54 \\
\hline 1000 & 441759.13 & 3681296.50 & 34.99 & 441759.41 & 3681296.52 & 34.78 \\
\hline $\mathrm{C} 1$ & 442181.34 & 3681837.57 & 36.89 & 442181.64 & 3681837.36 & 36.40 \\
\hline $\mathrm{C} 5$ & 442339.65 & 3681249.01 & 36.39 & 442339.31 & 3681248.94 & 36.39 \\
\hline C6 & 442482.30 & 3681346.87 & 36.10 & 442482.20 & 3681346.65 & 36.100 \\
\hline \multicolumn{7}{|c|}{ Mean Square Error in check points (m) } \\
\hline \multicolumn{2}{|c|}{ RMSE X $=0.3001$} & \multicolumn{2}{|c|}{ RMSE Y 0.1569} & & \multicolumn{2}{|c|}{ RMSE $Z=0.3527$} \\
\hline
\end{tabular}

Table 3. RMSE Delivered from Agisoft Photoscan Software.

\begin{tabular}{|c|c|c|c|c|c|c|}
\hline & \multicolumn{5}{|c|}{$\begin{array}{c}\text { Reference coordinates of check } \\
\text { Points (m.) }\end{array}$} & \multicolumn{2}{c|}{ Calculated coordinates of check Points } \\
\hline Point ID & X & Y & Z & X & Y & Z \\
\hline 1200 & 442024.99 & 3681031.63 & 35.71 & 442025.12 & 3681031.93 & 35.99 \\
\hline C9 & 441833.53 & 3681595.12 & 34.90 & 441833.64 & 3681595.05 & 35.17 \\
\hline C11 & 441846.08 & 3681868.01 & 36.15 & 441846.58 & 3681868.38 & 36.49 \\
\hline P9 & 442106.40 & 3682106.20 & 35.01 & 442106.19 & 3682106.07 & 34.49 \\
\hline C15 & 442103.99 & 3682051.10 & 35.20 & 442103.76 & 3682051.08 & 35.31 \\
\hline 1000 & 441759.13 & 3681296.50 & 34.99 & 441759.54 & 3681296.26 & 34.68 \\
\hline C1 & 442181.34 & 3681837.57 & 36.89 & 442181.89 & 3681837.14 & 37.09 \\
\hline C5 & 442339.65 & 3681249.01 & 36.39 & 442339.55 & 3681249.30 & 36.19 \\
\hline C6 & 442482.30 & 3681346.87 & 36.10 & 442482.23 & 3681347.19 & 35.88 \\
\hline \multicolumn{7}{|c|}{ Mean Square Error in check points (m) } \\
\hline \multicolumn{7}{|c|}{ RMSE X=0.310 RME Y =0.276 } \\
\hline
\end{tabular}



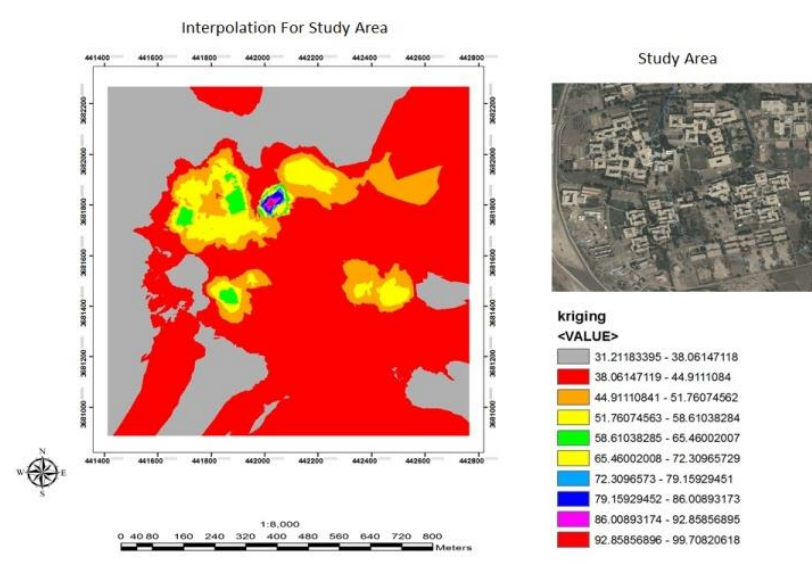

Fig.10. Interpolation by kriging Method (Results from LPS Software).
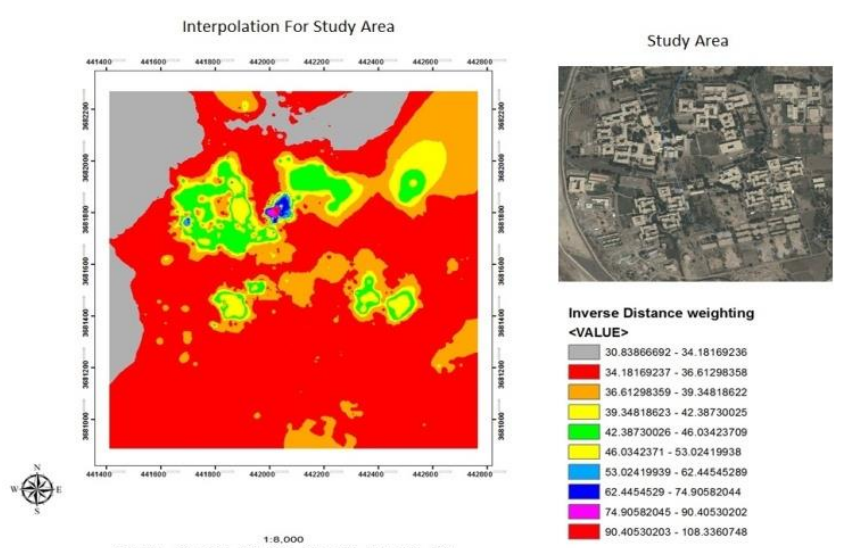

Fig.11. Interpolation by IDW Method (Results from LPS Software).

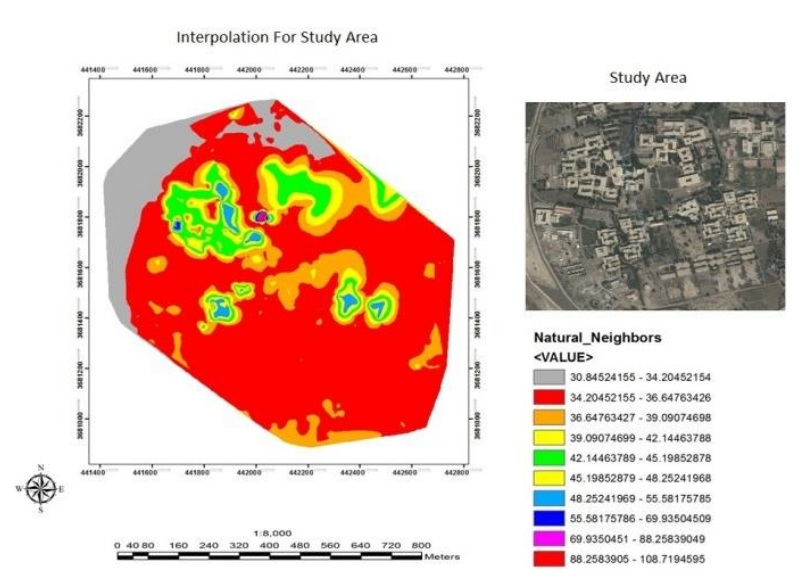

Fig.12. Interpolation by Nearest Neighbours Method (Results from LPS Software)

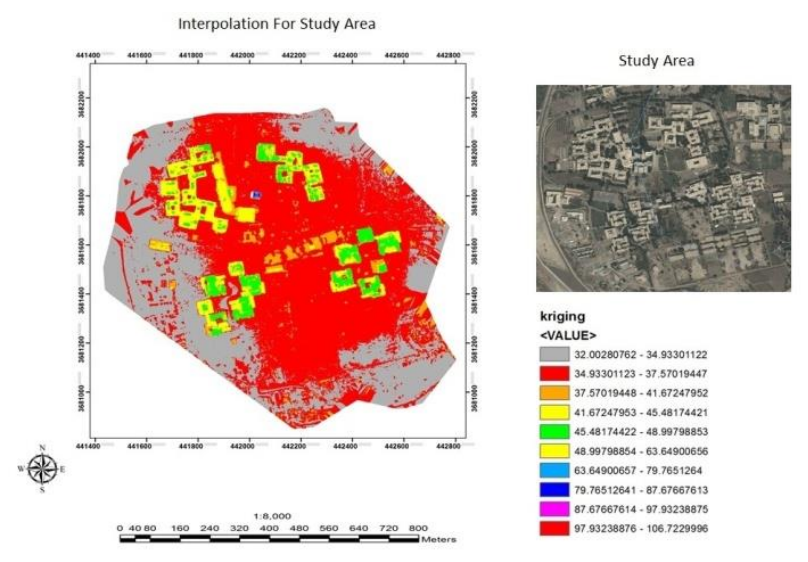

Fig.13. Interpolation by Kriging Method (Results from Agisoft PhotoScan Software).

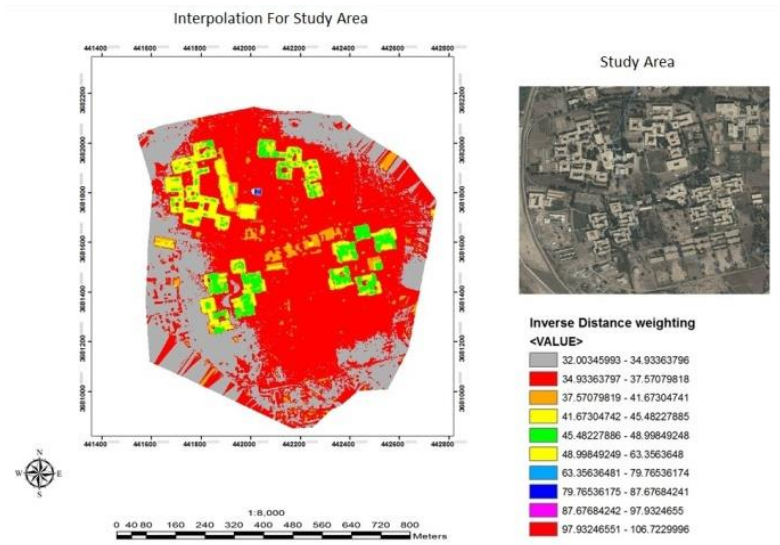

Fig.14. Interpolation by IDW Method (Results from Agisoft PhotoScan Software).

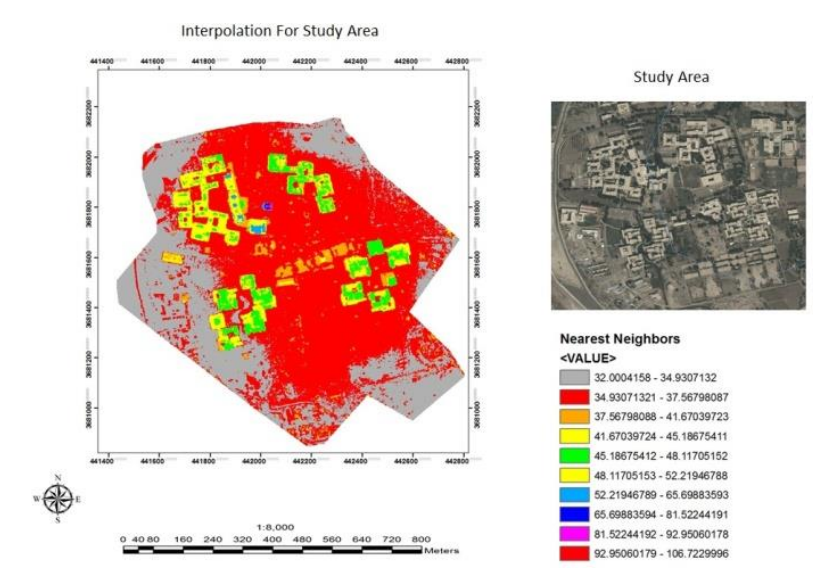

Fig.15. Interpolation by Nearest Neighbours Method (Results from Agisoft PhotoScan Software). 


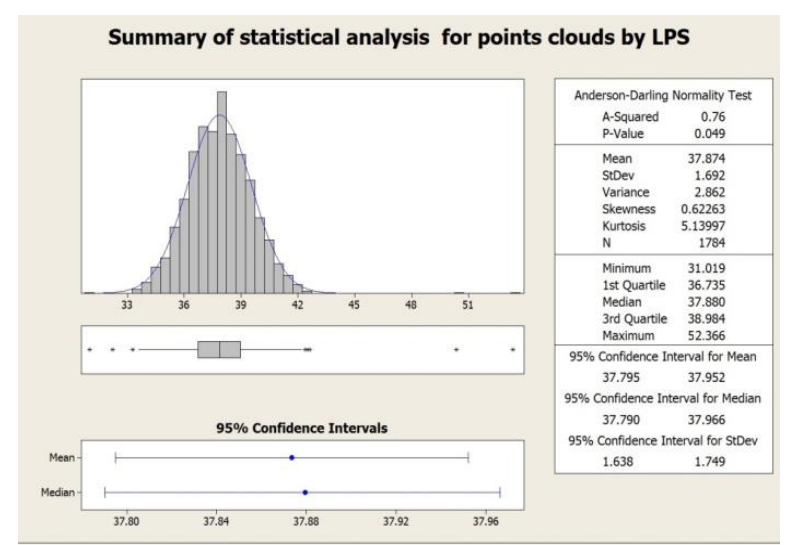

Fig.16. Summary Results of Statistical Analysis for LPS data.

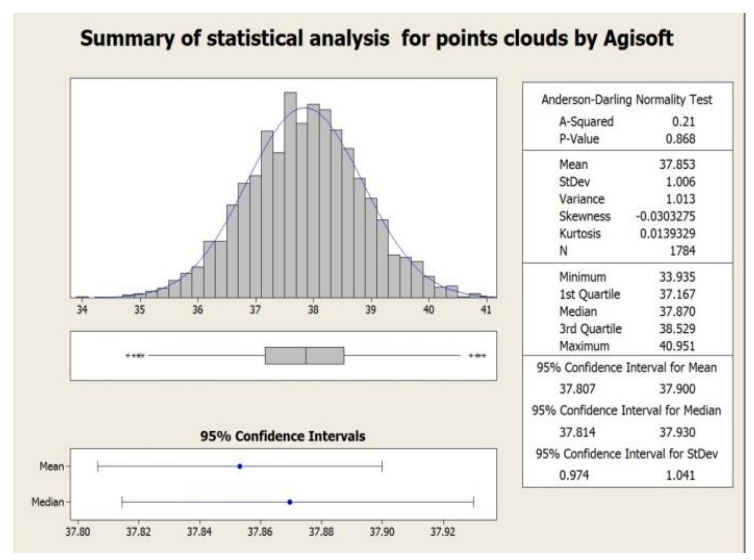

Fig.17. Summary Result of Statistical Analysis for Agisoft Photoscan data.

In order to evaluate the generated surfaces from the Agisoft Photoscan software, interpolated surfaces were assessed. Based on the standard deviation delivered, it was found that the kriging method is more reliable than other methods. Therefore, the Kriging method was selected to apply in the validation process with the reference measurements. The validation process was based on the shape geometric measurements for some accurate and generic objects, such as the length, width and the area of selected features. The results are shown in Tables 4 and 5 below.
Table 4. Illustrate the Validation Results of Lengths and Width Measurements.

\begin{tabular}{|c|c|c|c|c|c|}
\hline 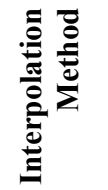 & 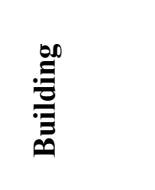 & $\frac{0}{0}$ & 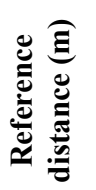 & 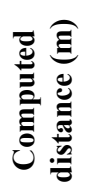 & $\underset{\mathscr{E}}{\widehat{\Xi}}$ \\
\hline & & $\mathrm{AB}$ & 70.31 & 72.43 & 2.12 \\
\hline & & $\mathrm{BC}$ & 64.22 & 62.12 & -2.1 \\
\hline & & $\mathrm{CD}$ & 48.09 & 46.17 & -1.92 \\
\hline & & $\mathrm{DE}$ & 49.97 & 51.18 & 1.21 \\
\hline & & EF & 20.97 & 20.7 & -0.27 \\
\hline & & $\mathrm{FG}$ & 58.23 & 59.11 & 0.88 \\
\hline & Building 1 & $\mathrm{GH}$ & 78.84 & 80.25 & 1.41 \\
\hline & & HI & 35.79 & 36.53 & 0.74 \\
\hline & & IJ & 48.08 & 49.97 & 1.89 \\
\hline & & JK & 58.2 & 56.54 & -1.66 \\
\hline & & KL & 69.75 & 70.94 & 1.19 \\
\hline & & LM & 36.18 & 33.85 & -2.33 \\
\hline & & MP & 13.98 & 12.79 & -1.19 \\
\hline & & PA & 114.61 & 111.41 & -3.2 \\
\hline Kriging & & & & & \\
\hline & & $\mathrm{AB}$ & 82.08 & 84.58 & 2.5 \\
\hline & Building 2 & $\mathrm{BC}$ & 58.22 & 60.6 & 2.38 \\
\hline & & $\mathrm{CD}$ & 82.08 & 84.59 & 2.51 \\
\hline & & DA & 58.22 & 60.69 & 2.47 \\
\hline & & $\mathrm{AB}$ & 52.13 & 55.5 & 3.37 \\
\hline & Building 3 & $\mathrm{BC}$ & 52.3 & 54.14 & 1.84 \\
\hline & & $\mathrm{CD}$ & 52.13 & 55.46 & 3.33 \\
\hline & & DA & 52.3 & 54.17 & 1.87 \\
\hline & & $\mathrm{AB}$ & 54.64 & 57.87 & 3.23 \\
\hline & Building 4 & $\mathrm{BC}$ & 54.97 & 56.96 & 1.99 \\
\hline & & $\mathrm{CD}$ & 54.64 & 57.89 & 3.25 \\
\hline & & DA & 54.97 & 56.97 & 2 \\
\hline & & & & \multicolumn{2}{|c|}{$\mathrm{SD}=2.234 \mathrm{~m}$} \\
\hline
\end{tabular}


Table 5. Illustrate the Validation Results of Area

\begin{tabular}{|l|c|c|c|c|}
\hline & Building & $\begin{array}{c}\text { Reference } \\
\text { area } \mathbf{~ m}^{\mathbf{2}}\end{array}$ & $\begin{array}{c}\text { Computed } \\
\text { area } \mathbf{~ m}^{2}\end{array}$ & $\begin{array}{c}\text { Diff } \\
\mathbf{m}^{\mathbf{2}}\end{array}$ \\
\hline & Building 1 & 13783.88 & 14094.54 & 310.67 \\
\hline Kriging & Building 2 & 4778.70 & 5130.27 & 351.58 \\
\hline & Building 3 & 2726.40 & 3035.08 & 308.68 \\
\hline & Building 4 & 3003.56 & 3320.92 & 317.35 \\
\hline \multicolumn{4}{|c}{ SD $=372.432 \mathrm{~m}^{2}$} \\
\hline
\end{tabular}

\section{Discussion and Conclusions}

In this paper, the ability of Agisoft PhotoScan software to derive reliable data from aerial images was assessed and analyzed accordingly and to subsequently show the potential of this software for accurate $3 \mathrm{D}$ modelling. To investigate this, a study was carried out in the University of Baghdad / Al-Jaderia campus using data collected from airborne metric camera with $457 \mathrm{~m}$ flying height. The Agisoft results show potential according to the research objective and the dataset quality following statistical and validation shape analysis. The reliability of the data delivered depends on different variables: the quality of the used imagery, image orientation, adopted scenario, GCPs availability and distribution, etc. The GCPs was measured and adjusted accurately following robust least squares scenario. The study is based on assessing the Agisoft results with those delivered from Leica Photogrammetric Suite software as a reference. Multiple interpolation methods were adopted, assessed and compared to find the most reliable surface created from the triangulated data delivered from the Agisoft software. Statistical analysis was applied and the results were interpreted accordingly for individual interpolation methods. Shape geometric measurements were conducted in the field following accurate and generic object selection in order to validate the generated 3D models. The following 3D RMSE values of $0.310 \mathrm{~m}$, $0.276 \mathrm{~m}$ and $0.294 \mathrm{~m}$ were delivered from Agisoft software. However, Leica Photogrammetry Suite has resulted $0.290 \mathrm{~m}, 0.157 \mathrm{~m}$ and $0.353 \mathrm{~m} 3 \mathrm{D}$ RMSE values in the $\mathrm{X}, \mathrm{Y}$, and $\mathrm{Z}$ respectively.

The results delivered indicate that the accuracy of the Aigesoft Photoscan software in the process of triangulation is high compared to the LPS software through the RMS outputs in terms of accuracy. From the methods of interpolation that depend on the data which was obtained from both softwares, it was found that interpolation results delivered from the Aigesoft Photoscan data is better than those delivered from the LPS data. This is because the number of points that intensified by the Aigesoft Photoscan are much more than the points intensified by LPS because of the ability of Aigesoft Photoscan to intensify the points during the process of triangulation to the number of millions of points contrary to the LPS software. Further, the results from the statistical analysis indicate that the method of kriging is the best interpolation method to intensify 3D points which is apparent from the standard deviation value delivered. Therefore, the following conclusions can be made upon the case study results:

$>$ The results show the reliability and the validation of Agisoft Photoscan software in the triangulation process and thus, in 3D modelling applications.

$>$ The great potential of Agisoft Photoscan software to intensify points during triangulation process in comparison with the LPS software for accurate 3D modelling applications.

$>$ The easy use of the Agisoft photoscan software comparing to the LPS software as the former doesn't need any approximate values to the unknowns including the exterior orientation parameters and the control points.

$>$ Kriging interpolation method is the best algorithm to generate surface from $3 \mathrm{D}$ data for accurate modelling.

\section{References}

1. A. Gruen, X. Wang, In Photogramm. Week (1999)

2. K. Hammoudi, Contributions to the $3 D$ city modeling: $3 D$ polyhedral building model reconstruction from aerial images and $3 D$ facade modeling from terrestrial $3 D$ point cloud and images (Université Paris-Est, 2011)

3. M. Lancelle, D.W. Fellner, In Image Vision Comput. 363, (2004)

4. R. Billen, et al. $3 D$ City Models and urban information: Current issues and perspectives (EDP sciences, 2014)

5. I. Suveg, G. Vosselman, ISPRS J. Photogramm. 58, 3 (2004)

6. N. Yastıklı, Z. Alkış, O. Emem, In CIPA XIX Conf. Proc. (2003)

7. Y. Al-kinani, Eng. Tech. J. 8, 33 (2015)

8. S. Singh, K. Jain, V. Mandla, In Geomatrix-12 Conf. Proc. (2012)

9. A. Rasam, et al. In 4th IEEE ICSGRC Conf. Proc. (2013)

10. A. Ali, C. Berebbia, Digital Architecture and Construction (WIT Press, 2006)

11. A. Abdul-rahman, S. Zlatanova, V. Coors, Innovations in 3D Geo Information Systems (Springer, 2006)

12. A. Aguilar, et al. In IMProVe Conf. Proc. (2011)

13. P. Wolf, B. Dewitt, Elements of photogrammetry: with applications in GIS (McGraw-Hill, 2000)

14. S. Ghosh, Photogrammetria. 31, 3 (1975)

15. I. Erdas, LPS project manager user guide (Erdas Inc., 2008)

16. L. Agisoft, Agisoft PhotoScan user Manual (Agisoft LLC, 2014)

17. C. Childs, E. Services, Arc user 32, 7 (2004)

18. C.. Yang, et al. In XX ISPRS Congress (2004) 
19. Y. Hu, Mapping monthly precipitation in Sweden by using GIS (Gothenburg, 2010)

20. R. Rossi, J. Dungan, L. Beck, Remote Sens. Environ 49, 1 (1994)

21. R. Webster, M. Oliver, Geostatistics for environmental scientists (John Wiley \& Sons, 2007) 